\title{
A Multi-ObJective Based Evolutionary ALgORITHM AND SOCIAL NETWORK ANALYSIS APPROACH FOR DYNAMIC Job SHOP SCHEDULING PROBLEM
}

\author{
${ }^{1}$ V.K.Manupati, ${ }^{2}$ N.Arudhra, ${ }^{2}$ P.Vigneshwar, ${ }^{2}$ D. RajaShekar and ${ }^{2}$ M.Yaswanth \\ ${ }^{1}$ Associate professor, Department of Mechanical engineering, KLUniversity, Vijayawada, \\ AP \\ ${ }^{2}$ Under Graduate Student, Department of Mechanical engineering, \\ KLUniversity,Vijayawada, AP
}

\begin{abstract}
In this paper, a multi-objective based NSGA-II algorithm is proposed for dynamic job-shop scheduling problem (DJSP) with random job arrivals and machine breakdowns. In DJSP schedules are usually inevitable due to various unexpected disruptions. To handle this problem, it is necessary to select appropriate key machines at the beginning of the simulation instead of random selection. Thus, this paper seeks to address on approach called social network analysis method to identify the key machines of the addressed DJSP. With identified key machines, the effectiveness and stability of scheduling i.e., makespan and starting time deviations of the computational complex NP-hard problem has been solved with proposed multi-objective based hybrid NSGA-ll algorithm. Several experiments studies have been conducted and comparisons have been made to demonstrate the efficiency of the proposed approach with classical multiobjective based NSGA-II algorithm. The experimental results illustrate that the proposed method is very effective in various shop floor conditions.
\end{abstract}

\section{KEYWORDS}

Social network analysis, NP hard, NSGA-II algorithm, Dynamic job-shop scheduling problem.

\section{INTRODUCTION}

Recent manufacturing has been emphasized on short product life cycles, variety of products with medium production. This can be achieved through current information and communication technologies (ICT) due to their rapid advancements. Job shop scheduling problem (JSP) attracts considerable attention over other production problems due to its varied applications in the field of manufacturing industries [1]. From the Literature it is well proved that JSP is the most difficult combinatorial optimization problems and it is well known as NP-hard problems [2]. It is necessary to understand the behavior of the manufacturing system under real-time events, such as random job arrivals, machine breakdowns, etc. The dynamic nature of the system can be called as dynamic JSP, which is essential for the handling real- world scheduling problems [3].

In real shop floor conditions the production orders are executed after the schedule, this brings large deviations to the original sequence. Thus, it is necessary to identify key machines at the

DOI: $10.5121 /$ ijci.2015.4207 
International Journal on Cybernetics \& Informatics (IJCI) Vol. 4, No. 2, April 2015

beginning of simulation rather than randomly generating them. Hence, uncertain situations in shopfloor such as machine break down, random arrival of jobs, etc can be taken care. A key machine[4], described various types of topologies and how these topologies affect the search space for exploiting the desired solution. In their work, authors have presented the descriptive statistics such as average distance, diameter, and distribution sequence of various topologies and found that the series of statistics directly affects the performance of the topologies. In this paper, we have identified an approach called social network analysis method (SNAM) that can capture the key machines from the available machines. This has been achieved with two folds: first, in DJSP the structure of the network has been achieved where interactions between different resources and their influence on the performance of the manufacturing system can be achieved. Whereas, with the flexibility of self-contained structure in collaboration networks obtained from the proposed SNAM and its descriptive statistics the performance of the system can be analyzed

[5]. Second, the functional properties such as centrality measures is identified in much better way [6].

In this article, we have conducted social network analysis for identifying the key machines and how this key machines effect the system performance i.e., efficiency and effectiveness of the scheduling. As the problem is multi-objective in nature, it is necessary to handle simultaneously. Thus, we proposed a multi-objective based evolutionary algorithm based NSGA-II for solving the problem to optimality. It was found that the proposed algorithm obtained lower make span in less computational time and less starting time deviations. Thus, we were able to develop and implement an efficient approach for improving the performance of the system.

The remainder of this paper is organized as follows. In section 2 , we describe a concrete example and its notations. In Section 3, we developed a mathematical representation of the proposed model and social network analysis based solution approach is presented. Section 4 explains the classical and proposed multi-objective based NSGA-II algorithms for solving the proposed problem. The experimentation with different test instances their results are introduced in section 5. In Section 6 the results and their discussions are detailed. The paper concludes with section 7 which suggests the directions of future work.

\section{Problem Description}

In this section, we consider a dynamic job shop scheduling problem in stochastic environments with random job arrivals, auxiliary resources constraints, machine beak-downs. To enhance the performance measures of the system such as makespan and starting time deviation, a mathematical model has been developed. Since the job arrivals at the system is dynamically flow over time thus it follows a closely to exponentially distributions. Consequently, the machine breakdown and repair timing also assumed as an exponential distribution. Thus, the mean time between failure (MTBS) and the mean time to repair (MTTR) are the two parameters that denote the breakdown level of the shop floor i.e., the percentage of time the machines have failures. Here, the job arrival rate has considered as passion distribution with certain job release policy. It is a case of mixed integer non linear programming (MINLP) model with multi-objectives. To meet the requirements from real-world production, an evolutionary algorithm approach with social network analysis has been implemented to solve the problem. The above mentioned problem makes several assumptions that are worth highlighting.

\subsection{Assumptions}

1. Each machine can process only one operation of any job at a time.

2. Job's setup times are sequence dependent. 
International Journal on Cybernetics \& Informatics (IJCI) Vol. 4, No. 2, April 2015

3. Jobs may have different arrival (ready) times.

4. Machine must not be interrupted except machine breakdown while processing a job.

5. Processing times of jobs are machine dependent.

6. Preemption and machine breakdown are allowed.

7. Processing times and numbers of operable machines are known in advance.

Table 1. The Experimental Condition

\begin{tabular}{|l|l|l|}
\hline Dimensions & Characteristic & Specifications \\
\hline Job shop & Size & 4 to 5 machines \\
& Machine break down level & 0.025 \\
& MTTR & 5 \\
& MTBF,MTTR & Exponential distribution \\
& Key machine & $\begin{array}{l}\text { Consider from social network } \\
\text { analysis }\end{array}$ \\
\hline Jobs & Random arrival & Poisson distribution \\
& Job arrival rate & $0.125,0.25 .0 .5,1$ \\
& The number of new jobs & $11,12,13,14,15,16,20$ \\
& Job release policy & Immediate \\
& Processing time of an operation & $41,10]$ \\
& Schedule frequency & \\
\hline Performance measure & Make span & \\
& Starting time deviations & \\
\hline
\end{tabular}

\subsection{Mathematical Model}

By allocating the above mentioned assumptions and notations, the problem is being formulated as the following fuzzy mixed-integer non-linear programming (FMINLP) model.

Objectives :

$$
\begin{aligned}
\operatorname{Min} Z_{1} & =\sum_{i=1}^{N} C_{i} \\
\operatorname{Min} Z_{2} & =\sum_{i=1}^{M}\left(S T_{i}-S T_{i}^{*}\right)
\end{aligned}
$$

Subjected to constraints :

$\sum_{\substack{i=1 \\ i \neq j}}^{N} \sum_{k=1}^{M} X_{i j k}=1 ; \forall j$

$\sum_{\substack{i=1 \\ i^{1} j}}^{N} X_{i j k}=Y_{j k} ; \forall j, k$

$\sum_{j=1}^{N} X_{i j k} \leq Y_{i k} ; i=1, \ldots, N$

$j \neq 1$

$c_{j}^{*}+A_{N}(1-X i j k) \geq \operatorname{Max}\left\{c_{i}^{*}+s_{i j k}, s_{j}\right\}+p_{j k} ; \forall i, j, k$

$c_{j}^{*}+A_{N}\left(1-z_{i j}\right) \geq \operatorname{Max}\left(c_{i}^{*}, s_{j}\right)+\sum_{k=1}^{M} p_{j k} Y_{j k} ; \forall i, j \in S_{r} \quad(i \neq j)$

$z_{i j}+z_{j i}=1 ; \forall i, j \in S_{r, i \neq j}$

$\sum_{k=1}^{M} X_{i j k} \leq z_{i j} ; \quad \forall i, j \in S_{r, i} \neq j$

$C_{i} \geq 0 ; T_{i} \geq 0 ; \forall i, X_{i j k}, Y_{i k}, Z_{i j} \in\{0,1\} ; \forall i, j, k$. 
International Journal on Cybernetics \& Informatics (IJCI) Vol. 4, No. 2, April 2015

The above mentioned objectives, i.e. minimization of total makespan $\left(Z_{1}\right)$ total starting time deviation $\left(Z_{2}\right)$ are given by Eqs. (1) - (2), respectively. Eq (3) assures that each job is only assigned to one position on a machine. Eq (4) suggests that if job $j$ is allotted to machine $k$, then it is adopted by another job indicating dummy job 0 . Constraint (5) specify that at most one job can immediately adopt the previously allotted job $i$ on machine $k$. Constraint (6) computes the completion time of job when it is processed instantly after job $i$ on machine. Constraint (7 and 8) ensures that if job $i$ and $j$ needs same operation, one must be finished earlier before starting the other. Constraint (9) gives the relation between $\mathrm{X}_{i j k}$ and $\mathrm{Z}_{i j}$. Eventually Eq (10) points the nonnegativity and integrality constraints.

\section{Social Network Analysis Model}

The method describes how the manufacturing execution data can be extracted and viewed as a network with nodes. Here, the data have been taken from the literature (Zhou et al. and Shao et al., 2010). Consequently, various tests have been conducted with SNAM to find different characteristics of the obtained network. In detail, the SNAM is categorised into two steps: (a) network modelling, and (b) network analysis and it is mentioned in the following sections:

\subsection{Network Modelling}

Network consists of set of nodes connected with ties indicating interaction [9]. This section presents how the manufacturing system execution data can be represented as networks. The modelling algorithms in Ucinet software package and for visualizing the obtained results are submitted to Netdraw software package. The obtained network from the affiliation matrix is called as collaboration network. This collaboration network is more interesting and meaningful than the simple network in terms of its characteristics, size, etc. The above procedure has been repeated for the remaining scenarios and obtained different collaboration networks. These collaboration networks are depicted in

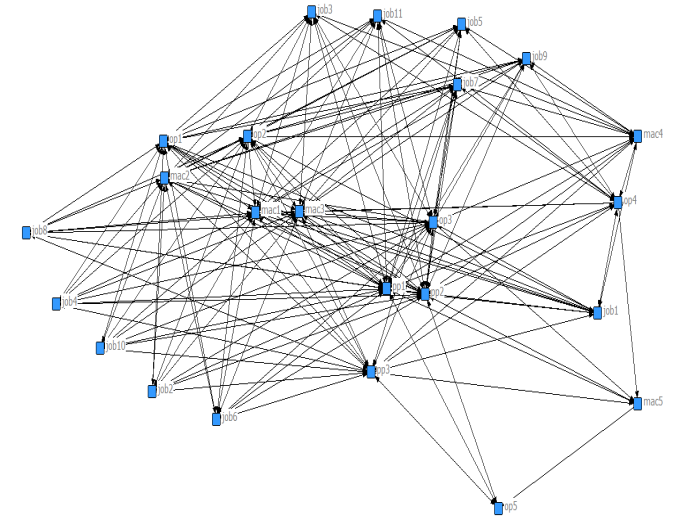

Figure 1. Random corroborative network

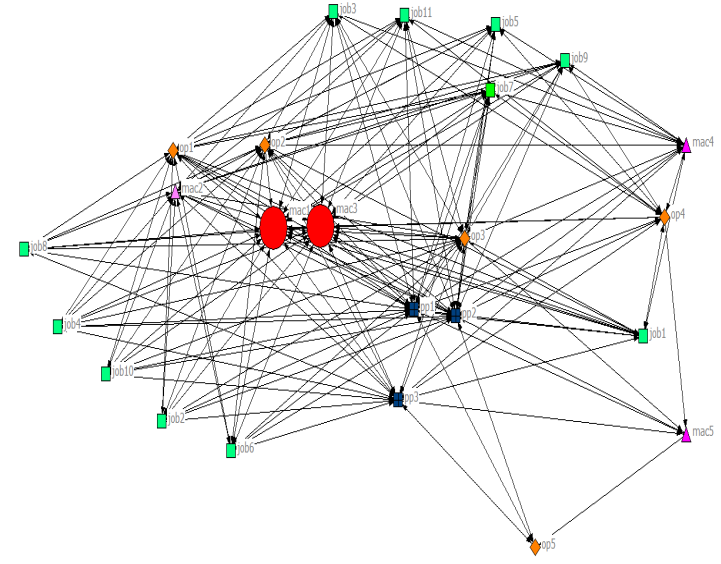

Figure 2. Corroborative network with key machines

\subsection{Centrality Measures of a Network}

Where, centrality is used to find how central a node is in the network. In this research, we have considered the two most popular centrality measures such as degree and betweenness centralities for the analysis of the network. The degree centrality measures the influence of the node from and to its closest neighbour with complexity of $\mathrm{O}(\mathrm{n})$ to linearly scale the nodes in the network, where $\mathrm{n}$ is the number of nodes. The second centrality measure, betweenness, has the higher level of 
International Journal on Cybernetics \& Informatics (IJCI) Vol. 4, No. 2, April 2015

control on the information floating between different nodes in the network [7]. In this paper, we have submitted the input data to the Ucinet and then obtained the results of degree and betweenness centrality of each attribute. In the below Table 2, the measures of three centralities of different instances of the particular work systems, are shown.

Table 2. Test Samples With Different Centralities

\begin{tabular}{|c|c|c|c|}
\hline $\begin{array}{l}\text { Instances } \quad \text { (jobs } \\
\times \text { machines) }\end{array}$ & Degree Centrality & Betweeness Centrality & $\begin{array}{l}\text { Identified key } \\
\text { machines from Social } \\
\text { network Analysis }\end{array}$ \\
\hline $11 \times 5$ & $\begin{array}{l}18 \\
16 \\
18 \\
12 \\
6\end{array}$ & $\begin{array}{l}8.310 \\
6.934 \\
8.310 \\
2.372 \\
0.742\end{array}$ & $\begin{array}{l}\text { Machine } 1 \\
\text { Machine } 3\end{array}$ \\
\hline $12 \times 5$ & $\begin{array}{c}16 \\
16 \\
16 \\
16 \\
8\end{array}$ & $\begin{array}{l}7.237 \\
7.237 \\
7.237 \\
7.237 \\
0.864\end{array}$ & $\begin{array}{l}\text { Machine } 1 \\
\text { Machine2 } \\
\text { Machine3 } \\
\text { Machine4 }\end{array}$ \\
\hline $13 \times 4$ & $\begin{array}{l}20 \\
20 \\
12 \\
11\end{array}$ & $\begin{array}{l}15.029 \\
15.029 \\
3.096 \\
4.129\end{array}$ & $\begin{array}{l}\text { Machine1 } \\
\text { Machine2 }\end{array}$ \\
\hline
\end{tabular}

\section{Multi-ObJective Nsga-Ii Algorithm}

In this research, we considered a multi-objective NSGA-II to generate optimal solutions. Where, Goldberg was the first who suggested the concept of non-dominated sorting genetic algorithm (NSGA) and Srinivas and Deb were the first to implement it. As NSGA differs from well known simple genetic algorithm is only in the way the selection operator works. Although NSGA has been used to solve a variety of MOPs, its main drawbacks include: (i) high computational complexity of non-dominated sorting, (ii) lack of elitism, and (iii) the need for specifying the tunable parameter. With the advent of NSGA-II, the above mentioned drawbacks have been resolved. We first apply a classical non-dominated sorting genetic algorithm-II (NSGA-II) [8] which includes Pareto ranking and crowding distance mechanism for selection of the individuals. Thereafter, with identified key machines from SNAM the MOEAs, has been implemented for solving the considered problem. The operators play a crucial role in the algorithm for generating of the better quality solutions. In order to find the better quality solutions, it is necessary to fine tune the parameters such as crossover and mutation in better manner. Therefore, the authors have said the ranges of operators i.e. crossover as 0.65 to 0.95 and mutation as 0.1 to 0.01 . By maintaining these ranges with the termination criteria as 100 iterations we have run the algorithm on an average of 10 per run. In this manner we improved the system performance better than the existing one. 


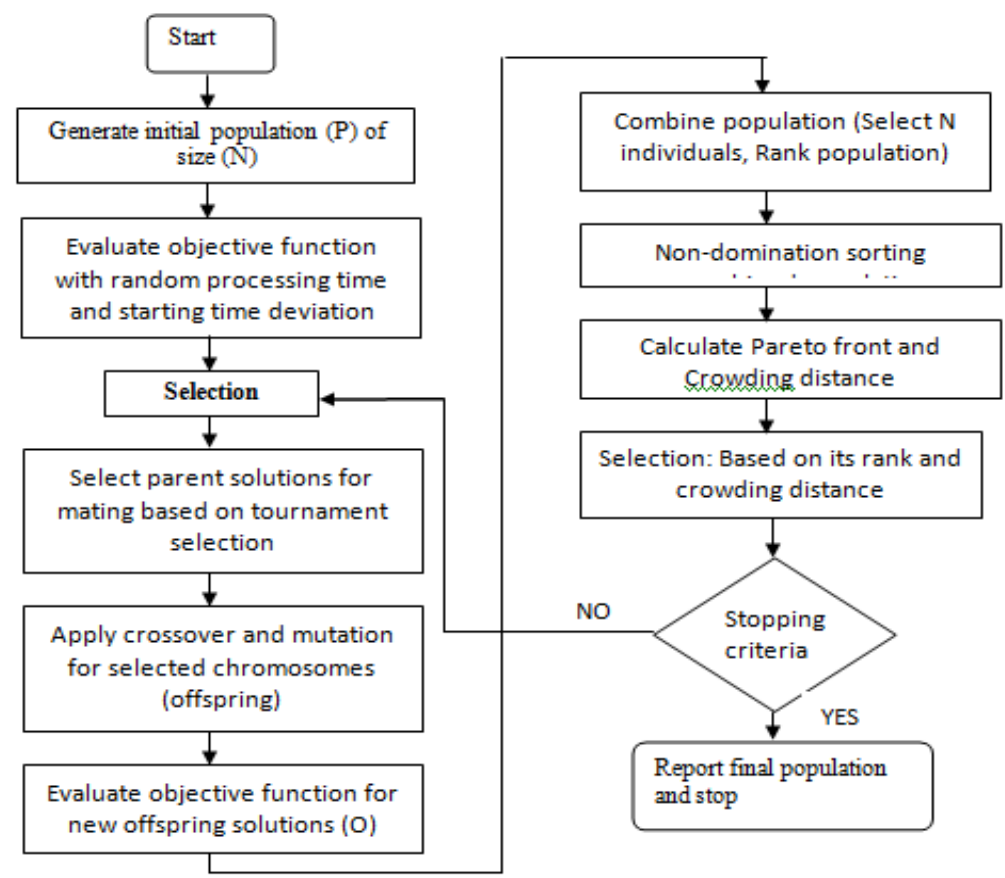

Figure 3.Schematic procedure for processing NSGA-II

\section{EXPERIMENTATION}

From Figure 4, the Pareto optimal solutions of the considered two performance measures with the classical NSGA-II and proposed NSGA-II algorithm is shown. Table 3 depicts different instances and their comparison results. For 11jobs 5 machines instance with classical NSGA-II algorithm the optimum makespan value is 28 , whereas for proposed NSGA-II it is 24 , similarly the starting time deviations for classical is 2 and proposed is 7 . This trend is continuing and the results are improving for proposed approach. All the mentioned algorithms are coded with MATLAB software and the problem is tested on Intel@ Core ${ }^{\mathrm{TM}} 2$ Duo CPU T7250 @2.00GHz, 1.99 GB of RAM.

Table 3. Comparison Of Different Instances With Classical Nsga-Ii And Proposed Nsga-Ii With Consideration Of Selected Key Machines

\begin{tabular}{|l|l|l|l|l|}
\hline $\begin{array}{l}\text { Instances } \\
\text { (Jobs } \\
\text { Machines) }\end{array}$ & \multicolumn{2}{|l|}{ NSGA- II } & \multicolumn{2}{|l|}{ NSGA-II with key machines } \\
\cline { 2 - 5 } & $\begin{array}{l}\text { Makespan } \\
\text { (Schedule } \\
\text { efficiency) }\end{array}$ & $\begin{array}{l}\text { Starting time } \\
\text { deviation (Schedule } \\
\text { stability) }\end{array}$ & $\begin{array}{l}\text { Makespan } \\
\text { (Schedule } \\
\text { efficiency) }\end{array}$ & $\begin{array}{l}\text { Starting time } \\
\text { deviation } \\
\text { (Schedule } \\
\text { stability) }\end{array}$ \\
\hline $11 \times 5$ & 28 & 2 & 24 & 7 \\
$12 \times 5$ & 37 & 1 & 32 & 3 \\
$13 \times 4$ & 42 & 2 & 36 & 4 \\
\hline
\end{tabular}


International Journal on Cybernetics \& Informatics (IJCI) Vol. 4, No. 2, April 2015

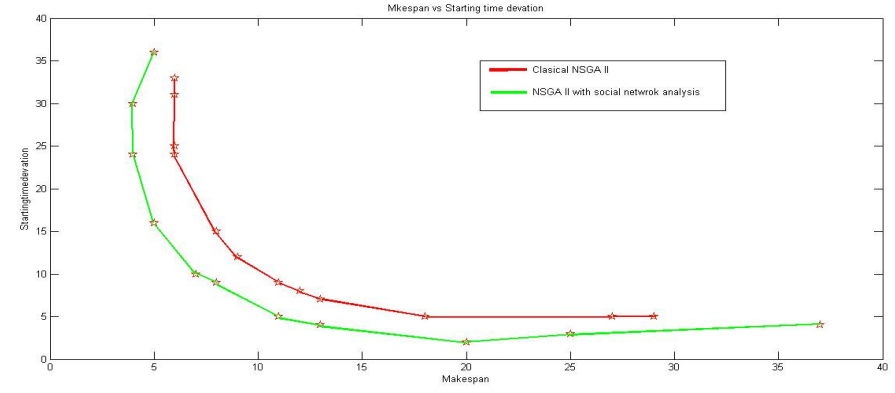

Figure 4. Comparison of classical NSGA II and proposed NSGA II with social network analysis

\section{Conclusions And Future Work}

This current paper elaborates in details of the proposed social network analysis method and multiobjective based NSGA-II evolutionary algorithm to solve the dynamic job shop scheduling problem. To find the performance of the proposed approach and to improve the performance of the manufacturing system two major performance measures such as scheduling efficiency i.e., makespan and scheduling effectiveness i.e., starting time deviations are measured. Hence, the job shop scheduling is computationally complex and well proved NP-hard in nature it is necessary to handle with evolutionary algorithm based approach. Here, we have identified an NSGA-II algorithm to solve the above mentioned problem. However, the identified problem is much complex than the general job shop because the uncertainity in job arrival and machine breakdown is considered one should take care of key machines identification. The proposed social network analysis method helps to identify the key machines dynamically thus the considered performance measure is enhanced. Moreover, with classical NSGA-II algorithm the proposed social network based evolutionary algorithm is compared. Experimental results shows that the proposed approach show its superiority over other. Further work may include generation of hybrid algorithms to solve on more problems with many performance measures that affect the system.

\section{REFERENCES}

[1] Kataev, Michael Yu, et al. "Enterprise systems in Russia: 1992-2012."Enterprise Information Systems 7.2 (2013): 169-186.

[2] Xia, Weijun, and Zhiming Wu. "An effective hybrid optimization approach for multi-objective flexible job-shop scheduling problems." Computers \& Industrial Engineering 48.2 (2005): 409-425.

[3] Ouelhadj, Djamila, and Sanja Petrovic. "A survey of dynamic scheduling in manufacturing systems." Journal of Scheduling 12.4 (2009): 417-431.

[4] Mendes, R., Kennedy, J., \& Neves, J. The fully informed particle swarm: simpler, maybe better. Evolutionary Computation, IEEE Transactions on, 8(3) 2004, 204-210.

[5] Wasserman, Stanley, and Joseph Galaskiewicz, "Advances in social network analysis: Research in the social and behavioral sciences". Vol. 171. Sage Publications (1994).

[6] Newman, Mark EJ. "Power laws, Pareto distributions and Zipf's law."Contemporary physics 46.5 (2005): 323-351.

[7] Hildorsson, Fredrik, and Tor Kvernvik. "Method and Arrangement for Supporting Analysis of Social Networks in a Communication Network." U.S. Patent Application 13/498,230.

[8] Srinivas, N., and Deb, K.. "Multi-objective optimization using nondominated sorting in genetic algorithms". Evolutionary Computation, 1995, 2(3), 221-248.

[9] Clauset, Aaron, Mark EJ Newman, and Cristopher Moore. "Finding community structure in very large networks." Physical review E 70.6 (2004): 066111. 
International Journal on Cybernetics \& Informatics (IJCI) Vol. 4, No. 2, April 2015

[10] Shao, Xinyu, et al. "Integration of process planning and scheduling-a modified genetic algorithmbased approach." Computers \& Operations Research 36.6 (2009): 2082-2096. 